\title{
Directional medium access control for ad hoc networks
}

\author{
Jianfeng Wang $\cdot$ Hongqiang Zhai $\cdot$ Pan Li $\cdot$ \\ Yuguang Fang $\cdot$ Dapeng Wu
}

Published online: 12 February 2008

(C) Springer Science+Business Media, LLC 2008

\begin{abstract}
Using directional antennas in ad hoc networks may introduce the well-known deafness problem, exacerbate the hidden terminal problem and the exposed terminal problem, add difficulty on mobile communication, and distort the operation of existing routing and TCP protocols. Although a lot of studies have been undertaken on the directional MAC protocols, most of them focus only on one or several aspects in their design and performance evaluations, and a comprehensive comparative study is missing. In this paper, we first explore the design space of directional MAC and present a taxonomy of existing schemes. Then, we discuss the major problems in using directional antennas under different category of MAC schemes. After that, we propose coordinated directional medium access control (CDMAC), a novel directional MAC protocol to improve throughput via facilitating the simultaneous contention-free communications for multiple local node-pairs. We evaluate our CDMAC, one representative existing scheme and IEEE 802.11 via extensive ns2 simulations. Our results show CDMAC provides a satisfactory solution
\end{abstract}

\author{
J. Wang · H. Zhai · P. Li · Y. Fang ( $) \cdot$ D. Wu \\ Department of Electrical \& Computer Engineering, University \\ of Florida, Gainesville, FL 32611-6130, USA \\ e-mail: fang@ece.ufl.edu \\ J. Wang \\ e-mail: jfwang@ufl.edu \\ H. Zhai \\ e-mail: zhai@ecel.ufl.edu \\ P. Li \\ e-mail: lipanleo@ufl.edu \\ D. $\mathrm{Wu}$ \\ e-mail: wu@ece.ufl.edu
}

to all the major problems and significantly improves the throughput performance.

Keywords Medium access control (MAC) . Ad hoc networks $\cdot$ Directional antennas

\section{Introduction}

Directional antennas technology offers a variety of potential benefits for wireless ad hoc networks. With directional transmission and reception, spatial reuse ratio and antenna gain can be increased substantially; this leads to significant improvement on communication efficiency.

However, using directional antennas may introduce the well-known deafness problem, exacerbate the hidden terminal problem and the exposed terminal problem, add difficulty on the neighbor discovery, and increase a lot of signalling overhead. Some of these problems not only affect the local communication efficiency but lead to ill operation of existing routing protocols (e.g., DSR and AODV) and transport protocols (e.g., TCP). For example, the deafness problem [11] may cause frequent false linkbreakage indication to the routing layer and destabilize the end-to-end congestion control.

A lot of directional MAC schemes [4-9, 11-15] have been proposed in recent years. However, most of them focus only on one or several aspects in their design and performance evaluation, and a comprehensive comparative study is missing. The primary contributions of this paper are as follows:

- We provide a first-principles approach to fully understanding the major MAC problems in using directional antennas. 
- We propose a novel directional MAC protocol called CDMAC to improve network throughput. A key feature of CDMAC is the introduction of a MAC timing structure to allow the node-pair which first captures the channel to locally coordinate multiple parallel (simultaneous) DATA/ACK transmissions, thus can dramatically increase the spatial reuse. Moreover, with the use of omni RTS/CTS, the deafness problem is almost eliminated and the hidden terminal problem is greatly alleviated with comparatively low signalling overhead.

- We present a thorough study of the end-to-end performance of the new protocol as a function of traffic, node density, mobility, and antenna gain.

The rest of the paper is organized as follows. In the next section, we describe the system model. Then we explore the design space and investigate the major design problems in Sects. 3 and 4, respectively. Section 5 presents our scheme. In Sect. 6, we evaluate our scheme, one representative existing scheme and IEEE 802.11 via ns2 simulation. Finally, we conclude the paper in Sect. 7.

\section{System model}

We consider CSMA/CA based MAC design for multihop wireless ad hoc networks. Each node has only one transceiver that transmits/receives signal in the same carrier frequency band. Assume single-beam directional antenna, particularly the switched beam antenna, is equipped in each node, which can generate one high-gain main-lobe beam in a particular direction together with several low-gain sidelobe beams in other directions. As a widely used assumption, each node can run in two operational modes, i.e., omnidirectional mode and directional mode. In other words, each node can dynamically switch between omnidirectional transmission/reception and directional transmission/reception. When a node is in idle state, it runs in omnidirectional mode to receive signal.

Directional antennas basically provide two folds of potential benefits: high spatial reuse and high antenna gain. Assume the number of antenna element is $M$. The maximal spatial reuse ratio, which is interpreted as the number of concurrent DATA transmissions in a local area, can be as high as $M$ in an LOS environment, even each participating node is in the omnidirectional transmission range of others. Another benefit of using directional transmission and reception is the increase of the antenna gain. Denote the main-lobe antenna gain as $G^{d}$ and the side-lobe antenna gain as $G^{S}$ when antenna runs in directional mode for either transmitting or receiving. Denote the omnidirectional gain as $G^{o}$ when antenna runs in omnidirectional mode for either transmitting or receiving. Typically, $G^{d} \geq G^{o} \gg G^{s}$.

\section{Objectives, design space and existing solutions}

The directional MAC design objectives consist of at least the following aspects: (1) to maximally utilize spatial reuse and directional antenna gain; (2) to work friendly with existing routing protocols (including the neighbor discovery) and transport protocols which have been developed and/or tuned based on omnidirectional medium access control; (3) to maintain backward compatibility with IEEE 802.11 whenever possible, which means that an 802.11 node can transparently communicate with other directional-antennas-capable nodes; (4) to support high mobility; (5) to keep signalling overhead low.

Next, we explore the design space and classify some existing solutions and our suggested solution.

\subsection{Design space}

We limit our design space to the framework in which contention-based RTS/CTS (control message) precede DATA/ACK (data transmission and acknowledgement) to probe receiver, bootstrap beamforming and reserve channel. However, the solution under this framework could be varied according to the RTS/CTS handshake rule and the MAC timing structure of RTS/CTS + DATA/ACK.

\subsubsection{RTS/CTS handshake}

Two basic RTS/CTS handshake rules were investigated in the literature. One is omni RTS/CTS (denoted as O-RTS/ O-CTS) [4, 5]; the other is directional RTS/CTS (denoted as D-RTS/D-CTS) [7, 8, 15]. As shown in Fig. 1, these two handshake rules will lead to much different RTS/CTS coverage. The beamwidth of directional RTS/CTS, defined as $\theta$, can be approximated as $360^{\circ} / M$. The transmission range $r$ can be represented as a function of antenna gain $\left(G_{t}\right.$ and $G_{r}$ ) as well as other factors [2]:

$r=\left(\frac{P_{T} G_{t} G_{r}}{K\left(I+\sigma^{2}\right) \gamma}\right)^{1 / \alpha}$,

where $P_{T}$ is the transmission power, $K$ is a constant that accounts for absorption, ohmic losses, etc., $I$ is interference, $\sigma^{2}$ is noise strength, $\gamma$ is the threshold of the signalto-interference-plus-noise ratio to support specific transmission rate (higher transmission rate requires higher $\gamma$ ), $\alpha$ is the path loss factor (which normally ranges from 2 to 5). Considering RTS/CTS is transmitted directionally and 

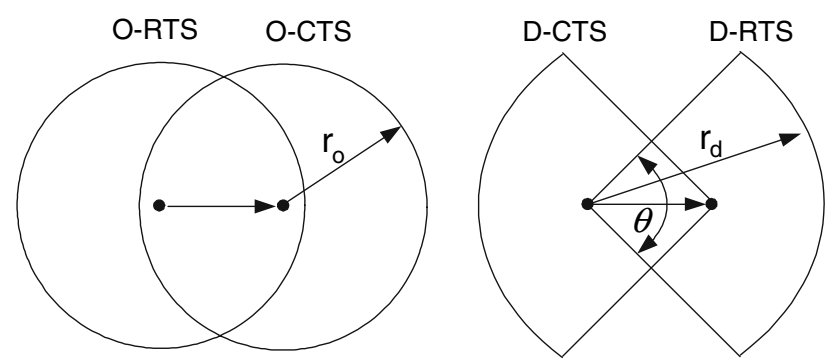

Fig. 1 RTS/CTS coverage

received omnidirectionally, $r_{d}$ (the transmission range under directional transmission) can be $\left(G^{d} / G^{o}\right)^{1 / \alpha}$ times of $r_{o}$ (the transmission range under omni transmission), if other parameters are the same.

To be explained in Sect. 4, the larger the RTS/CTS coverage, the less the deafness problem, the less the hidden terminal problem (assuming the RTS/CTS coverage is enlarged without increasing the transmission power of RTS/CTS), and the less problem to identify a mobileneighbor's location.

In addition to the two basic RTS/CTS handshake rules, we note the circular directional RTS/CTS (denoted as Circular D-RTS/D-CTS) was also suggested to increase the RTS/CTS coverage as much as possible $[9,10]$. The downside of circular RTS/CTS scheme is the high signalling overhead.

\subsubsection{MAC timing-structure}

Two basic MAC timing-structures were presented in the literature for communication among multiple local nodepairs; one is denoted as 802.11 MAC timing-structure, in which a node-pair's DATA/ACK strictly follows the nodepair's RTS/CTS; the other is coordinated MAC timingstructure, in which multiple RTS/CTSs exchanged among multiple local node-pairs are allowed to take place to reserve channels, one for each node pair, before a coordinated period for DATA/ACK transmission. All the existing directional MAC solutions use the 802.11 MAC timingstructure. The coordinated MAC timing-structure was developed to alleviate the exposed terminal problem in omnidirectional-antennas based ad hoc networks [16, 17], the application of which to the ad hoc networks using directional antennas has not been investigated yet.

The MAC timing-structure together with RTS/CTS handshake rule affects spatial reuse. With IEEE 802.11 MAC timing-structure, larger RTS/CTS coverage leads to lower spatial reuse. However, with coordinated timingstructure, sufficiently large RTS/CTS coverage actually helps the spatial reuse rather than hurts the spatial reuse.

\subsection{Classification of solutions}

According to the RTS/CTS handshake rule and the timingstructure of RTS/CTS + DATA/ACK, we may classify existing solutions and our proposed solution into four categories, as shown in Fig. 2. The D-DATA and D-ACK in the figure denote the directional data transmission and the directional acknowledgement, respectively. The first three categories of solutions have been presented in the literature. The category I, which includes $[4,5]$, uses O-RTS/O-CTS + 802.11 MAC timing-structure. The category II, which includes [7, 8, 15], uses D-RTS/D-CTS + 802.11 MAC timing-structure. The category III, which includes [9], uses circular D-RTS/D-CTS + 802.11 MAC timing-structure. The category IV, which we are going to investigate in this paper, uses O-RTS/O-CTS/O-CRTS + coordinated MAC timing structure; O-CRTS here represents the omni confirmed-RTS (CRTS), a short message used to confirm the reservation of spatial channel, which may not be necessary in single-hop case but helps in multihop ad hoc networks (see more details in Sect. 5).

\section{Problem and observation}

In this section, we discuss the major open problems in the directional MAC design. These problems include the deafness problem, the hidden terminal problem, the exposed terminal problem, and the distant/mobile neighbor communication problem. We will identify the origin of each problem (which may exist in one or several or all the existing solutions), evaluate its impacts on the network performance and discuss how our suggested solution can help alleviate or eliminate the problem.

\subsection{Deafness problem}

The deafness problem can be defined in various ways but in this paper it specifically refers to the problem arises when an intended transmitting node fails to communicate with an intended receiving node because the intended receiving node is beamformed in a direction away from the intended transmitting node [8, 11]. For example, as shown in Fig. 3, node A senses channel is idle and tries to send RTS directionally to node B. However, node B is currently communicating with node $\mathrm{C}$ by beamforming towards $\mathrm{C}$. Since node B cannot reply CTS to node A, node A may falsely think RTS is collided or think node B has moved to another position; if node A assumes RTS is collided, node A may keep transmitting RTS until it succeeds or the retransmission number reaches the maximal retry limit; if node A assumes node B has moved, node A may initiate 
Fig. 2 Time-diagram of multiple RTS/CTS/DATA/ACK

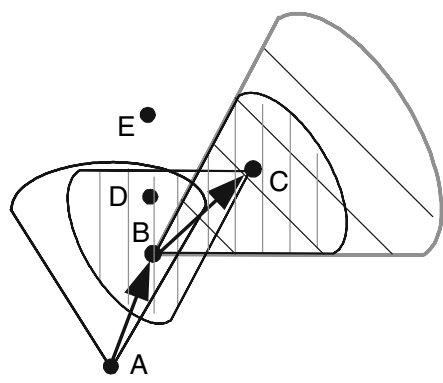

Physical Carrier

Sensing Region

Directional RTS/CTS

Coverage

Fig. 3 An illustration of deafness problem

the neighbor-discovery mechanism to locate node $\mathrm{B}$ before sending RTS again to node B or may initiate re-routing procedure.

We note there is no such deafness problem in IEEE 802.11 when RTS/CTS and DATA/ACK are transmitted omnidirectionally. The deafness problem is introduced by using directional antennas, particularly, by the directional transmission of RTS/CTS. To be clear, we refer to Fig. 3 for explanation. In Fig. 3, due to the directional transmission of RTS/CTS, the RTS/CTS coverage is limited to the "II" shadowing area. Since node A is outside of the RTS/ CTS coverage and the physical carrier sensing region, node $A$ is not aware of communication between node $B$ and node $\mathrm{C}$, thus may initiate transmission to B.

We note that a node in the RTS/CTS coverage could also cause deafness problem. For example, node D in Fig. 3 was being beamformed towards node $\mathrm{E}$ when node $\mathrm{B}$ and node $\mathrm{C}$ exchanged RTS/CTS. After node D completes communication with $\mathrm{E}$, node $\mathrm{D}$ wants to communicate with node B even though node B is transmitting data to $\mathrm{C}$, thus causing deafness problem.

The deafness problem can be so severe that it may totally offset the advantages of using directional antennas if left unaddressed [11]. The negative effects of deafness problem are as follows.

- The deafness problem will waste energy and network capacity as a result of network nodes engaging in repeated, unproductive transmissions.

- The deafness problem will cause the ill behavior of a collision resolution algorithm because an intended transmission node cannot tell the reason of transmission failure, which may be due to collision or due to the deafness problem.

- The deafness problem may cause false link-breakage indication to the routing layer and result in unnecessary re-routing. According to AODV or DSR, a link is considered broken when the number of transmission failure is larger than certain threshold.

- The deafness problem may cause end-to-end congestion control more unstable.

The deafness problem can be alleviated significantly if each node always transmit RTS/CTS omnidirectionally and each of its neighbors hearing RTS/CTS is not allowed to contend for channel during other ongoing DATA/ACK transmissions. Unfortunately, the spatial reuse will be hurt significantly if we use omni RTS/CTS while still following the 802.11 timing-structure [5]. In other words, there exists a tradeoff between the deafness problem and the spatial reuse under the 802.11 MAC timing-structure. However, there is no such tradeoff if we use coordinated MAC timing-structure together with omni RTS/CTS; that is to say, we can alleviate or totally remove the deafness problem while still keeping high spatial reuse.

We note Choudhury and Vaidya extensively studied the negative effects of deafness problem in [11] and proposed a mechanism named out-of-band tone to alleviate the problem. But the out-of-band tone solves only part of the 
deafness problem yet requires channel splitting and more complex transceiver, it will be more desirable if we can solve the problem using "in-band" solution and solve the problem more completely.

\subsection{Hidden terminal problem}

To be general, we define a hidden terminal as a terminal which is not aware of another node-pair's ongoing communication but whose intended transmission can make another node-pair's communication unsuccessful.

Hidden terminal has already been a severe problem in omnidirectional communication. When it comes to the directional communication, especially if RTS/CTS is transmitted in the directional manner, the hidden terminal problem becomes harder to address [8]. Unlike the omnidirectional communication, a node in RTS/CTS coverage could also be the hidden terminal. According to whether a hidden terminal is in the RTS/CTS coverage, we define Type I hidden terminal as the hidden terminal in the RTS/ CTS coverage and define Type II hidden terminal as the hidden terminal outside the RTS/CTS coverage.

Type I hidden terminal problem is resulted from the directional transmission of RTS/CTS/DATA/ACK. An RTS/CTS will not be heard by a node in the RTS/CTS coverage when the node was being beamformed away from the node-pair which exchanged the RTS/CTS. For example, in Fig. 4, node D was being beamformed towards node E when node $\mathrm{B}$ and node $\mathrm{C}$ exchanged RTS/CTS. After node $\mathrm{D}$ completes communication with node $\mathrm{E}$, node $\mathrm{D}$ wants to communicate with node $\mathrm{C}$ even though node $\mathrm{C}$ is receiving data from node $\mathrm{B}$, thus causing collision at node C. Type II hidden terminal problem is due to the gap between the transmission range of RTS/CTS and the interference range. For example, in Fig. 4, node $\mathrm{A}$ is out of the RTS/CTS coverage so that node A will not be aware of the transmission from node B to node C. But node A's intended transmission will cause collision at node $\mathrm{C}$.

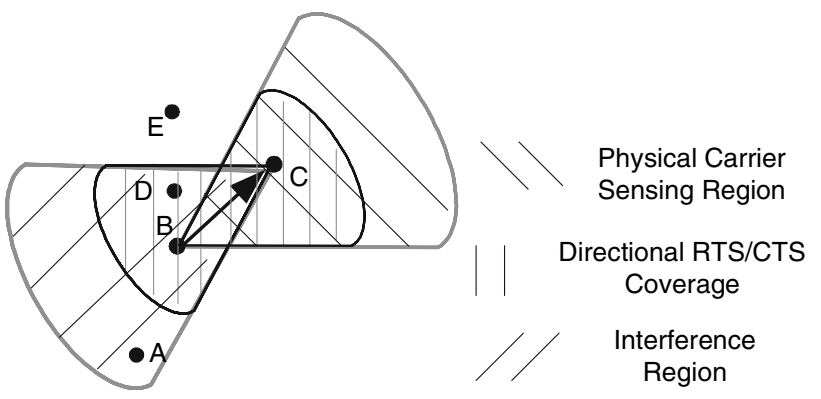

Fig. 4 An illustration of hidden terminal problem
To alleviate or totally eliminate the Type I hidden terminal problem, we should use omnidirectional transmission of RTS/CTS rather than directional transmission of RTS/CTS. As we know, to avoid reducing the spatial reuse, coordinated MAC timing-structure rather than 802.11 MAC timing-structure should be used together with omni RTS/CTS. To alleviate the Type II hidden terminal problem, we need to reduce the gap between the transmission range of RTS/CTS and the interference range of DATA/ ACK. It will be very interesting if we can increase the transmission range of RTS/CTS without increasing the transmission power of RTS/CTS; for example, as suggested in Eq. 1, we can reduce the transmission rate of RTS/CTS to extend the transmission range of RTS/CTS without increasing the transmission power of RTS/CTS.

\subsection{Exposed terminal problem}

Exposed terminal problem is a problem in which two nodepairs are forbidden to transmit DATA simultaneously even though simultaneous data transmissions and simultaneous ACK transmissions of two node pairs will not collide with each other. For example, as shown in the Fig. 5(a), suppose node $\mathrm{A}$ intends to transmit DATA to $\mathrm{B}$ and exchanges RTS/CTS in the first place. At the same time, node $\mathrm{C}$ wants to transmit DATA to D. Obviously, node A and node $\mathrm{C}$ can simultaneously transmit DATA without collision with each other; after both node A and node C complete DATA transmission, node $\mathrm{B}$ and node $\mathrm{D}$ can simultaneously transmit ACK without collision with each other either. However, if we follow the 802.11 MAC timing-structure, node $\mathrm{C}$ should defer its transmission after it hears the RTS/ CTS sent by node-pair AB; otherwise, the CTS to be sent by node $\mathrm{D}$ may collide with the DATA being received at $\mathrm{B}$, and so on. The exposed terminal problem significantly reduces the spatial reuse.

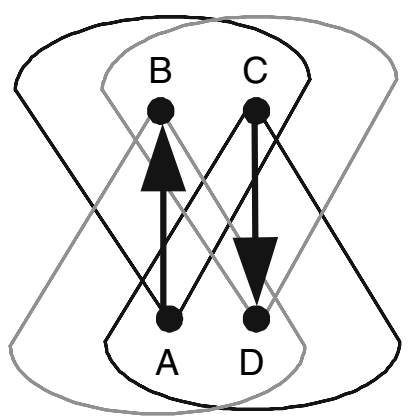

(a)

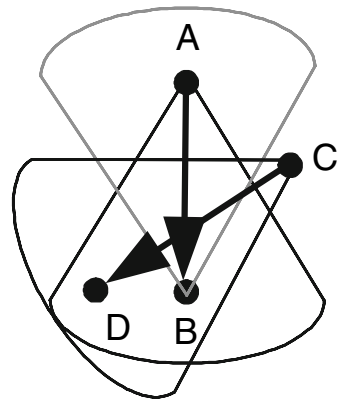

(b)
Fig. 5 (a) Exposed terminal problem; (b) Joint exposed terminal problem and receiver blocking problem 
Here we need to point out another type of exposed terminal problem; we name it as the joint exposed terminal problem and receiver blocking problem. As shown in Fig. 5(b), there is no collision if node-pair AB and node pair CD transmit data simultaneously and then transmit ACK simultaneously. However, if we use 802.11 MAC timingstructure together with directional RTS/CTS and assume each node in the idle state runs in the omni mode to receive signal, node D cannot reply CTS to node C if node A transmit RTS (then data after receiving CTS from node B) in the first place to node B. The joint exposed terminal problem and receiver blocking problem not only reduces spatial reuse but leads to the same negative effects as the deafness problem (explained in the previous section). Since node $\mathrm{C}$ is not aware of local communication activity, it will keep sending RTS to node D even though node D is blocked by the communication between node $\mathrm{A}$ and node $\mathrm{B}$.

It is fair to say that the 802.11 MAC timing-structure together with directional RTS/CTS results in severe exposed terminal problem. However, if we use our suggested coordinated MAC timing-structure plus omni RTS/ CTS, the exposed terminal problem will be much alleviated.

\subsection{Side-lobe problem}

Side lobes are portions of the electromagnetic response pattern of an antenna that is not contained in the main beam of a directional antenna. The side-lobe problem can arise if one node senses the RTS/CTS exchange of a pair of other nodes and decides that, since its own anticipated transmission to yet another node will not collide with the beam of the communicating node-pair, it can transmit a collisionfree signal to the other node. The problem occurs if the deciding node is in such close proximity to one of the two communicating nodes that a side lobe of the signal from the deciding node interferes with the signal between the communicating nodes.

For example, as shown in Fig. 6(a), node $\mathrm{C}$ can hear RTS/CTS exchange between node A and node B and knows

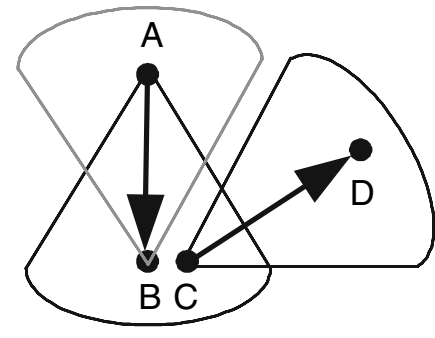

(a)

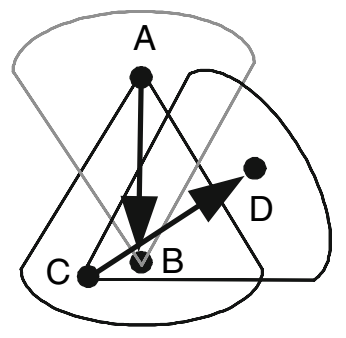

(b)
Fig. 6 An illustration of side-lobe problem: (a) Case 1; (b) Case 2 node A is transmitting DATA to node B. Simply because the transmission from node $\mathrm{C}$ to node $\mathrm{D}$ does not share the same beam-direction with that used for transmission from node $\mathrm{A}$ to node $\mathrm{B}$, node $\mathrm{C}$ thinks its intended transmission to node $\mathrm{D}$ will not cause collision. Thus, node $\mathrm{C}$ may begin to transmit RTS to node D or reply CTS to node D if node D initiates a RTS to node C. Unfortunately, since node $\mathrm{C}$ is so close to node $\mathrm{B}$, the side lobe of transmitting node $\mathrm{C}$ interferes the reception of node $\mathrm{B}$ (the interference power received at node $\mathrm{B}$ equals $\frac{P_{T} G^{s} G^{s}}{K r^{\alpha}}$ ); in principle, if the distance between node $\mathrm{B}$ and $\mathrm{C}$ is smaller than a certain threshold, any transmission of node $\mathrm{C}$ in arbitrary direction will ruin the DATA reception of node $B$ from $A$. The side lobe problem can be more serious if the node $B$ is in the main lobe node $C$, as shown in Fig. 6(b), where the interference power received at node B turns to be $\frac{P_{T} G^{d} G^{s}}{K r^{\alpha}}$, much higher than $\frac{P_{T} G^{s} G^{s}}{K r^{\alpha}}$ under the case shown in Fig. 6(a).

The side-lobe effect largely depends on how large sidelobe beam could be in comparison with the main-lobe beam. In reality, the side-lobe may not be too small to be ignored. The side-lobe problem has not been well addressed in the literature.

A possible solution for side-lobe problem is to add a side-lobe detection step implicitly or explicitly during channel contention and collision avoidance, specifically, during the exchange of RTS/CTS. We present the detailed method to alleviate the side-lobe problem in Sect. 5.

\subsection{Distant/mobile neighbor communication problem}

Denote the antenna method used for exchanging packets as no beamforming (N-BF), transmit beamforming ( $\mathrm{T}$ $\mathrm{BF}$ ), or transmit and receive beamforming (TR-BF) [15]. The use of term "beamforming" indicates the use of a directional antenna. Therefore, "no beamforming" means that the transmitter and the receiver use omnidirectional antennas, while "transmit beamforming" means that the transmitter uses a directional antenna, but the receiver uses an omnidirectional antenna, and so on. If the transmission power of directional transmission is allowed to be the same as that for omnidirectional transmission, the transmission range by $\mathrm{TR}-\mathrm{BF}$ will be greater than that under T-BF; similarly, the transmission range by $\mathrm{T}-\mathrm{BF}$ will be greater than that under N-BF. We define three types of neighbor according to the distance: the neighbor in N-BF transmission range (named N-BF neighbor), the neighbor outside the $\mathrm{N}-\mathrm{BF}$ transmission range but in the $\mathrm{T}-\mathrm{BF}$ transmission range (named $\mathrm{T}-\mathrm{BF}$ neighbor), and the neighbor outside the $\mathrm{T}-\mathrm{BF}$ transmission range but within the TR-BF transmission range (named TR-BF neighbor). We call both T-BF neighbors and TR-BF neighbors as distant neighbors. 
Table 1 Comparative study of four categories of solutions

\begin{tabular}{lllll}
\hline & Category I & Category II & Category III & Category IV \\
\hline Deafness problem & No & Severe & Medium & No \\
Type I hidden terminal problem & No & Severe & Medium & No \\
Type II hidden terminal problem & Medium & Medium & Medium & Light \\
Exposed terminal problem & Severe & Medium & Medium & Light \\
Difficulty for mobile-node communication & Light & High & Medium & Light \\
Signalling overhead & Light & Light & High & Medium \\
\hline
\end{tabular}

It is challenging for a node to directly communicate with its TR-BF neighbor because beamforming to each other is required before they can directly handshake, which is really hard under the context of contention based MAC. We note a mechanism named multihop RTSs [8] has been proposed to solve this problem (if there are intermediate nodes between a node and its TR-BF neighbor), but the complexity and overhead are serious issues. For communication between a node and its T-BF neighbor, it will be a little bit easier; the tradeoff is that we need to use directional RTS/CTS. As we said before, directional RTS/CTS will introduce serious deafness problem and aggravate the hidden terminal problem.

Moreover, in highly mobile environment, a neighbor may easily move from one beam direction to another, which could cause directional RTS to "mis-hit". The probability of "mis-hit" increases as the mobility increase or as the beamwidth used for sending RTS decreases.

Since the communication between two distant neighbors is hard, it may not be a good choice to directly communicate with the distant neighbors unless we really need, specifically, to maintain network connectivity. Instead of taking the advantage of directional antenna gain to increase onehop transmission range but with low data rate and same power, we can enjoy the high data rate or low transmission power with the same transmission range as omni case [19], which means we use N-BF neighbors for relaying packets but transmitting in higher rate. If we still use N-BF neighbors, we can use omnidirectional RTS/CTS instead of directional RTS/CTS to reach the neighbor, while avoiding the deafness problem. Furthermore, to overcome mobile neighbor communication problem, omni RTS/CTS will be a better choice, to reduce the probability of "mis-hit".

\subsection{Summary of some important observations}

Based on what we have discussed in the above, we get the following key observations.

Observation 1 Directional RTS/CTS will introduce deafness problem and Type I hidden terminal problem and complicate the communication between mobile nodes; Omni RTS/CTS will not introduce deafness problem and
Type I hidden terminal problem, and facilitate the communication between mobile neighbors.

Observation 2 802.11 MAC timing-structure limits spatial reuse significantly by leaving exposed terminal problem unaddressed; Coordinated MAC timing-structure can solve the exposed terminal problem and improve the spatial reuse substantially.

Observation 3 The direct communication between two distant neighbors is hard, thus is not desired if there are intermediate nodes can help relay; the directional antenna gain is preferably used for reducing the transmission power or increasing transmission rate, rather than reaching distant neighbors.

Observation 4 To alleviate Type II hidden terminal problem, we need to reduce the gap between the interference range and virtual carrier sensing range; an interesting solution is to apply low transmission rate and strong channelerror coding to RTS/CTS; which may increase the transmission time of RTS/CTS but will increase the transmission range of RTS/CTS without increasing transmission power.

The comparative study for 3 categories of existing solutions and our suggested solution is summarized in Table 1. To refresh the memory, Category I solution is characterized as omni RTS/CTS + 802.11 MAC timingstructure [4, 5]; Category II solution is characterized as directional RTS/CTS + 802.11 MAC timing-structure [7, 8, 15]; Category III solution is characterized as circular directional RTS/CTS + 802.11 MAC timing-structure [9]; Category IV solution is characterized as omni RTS/ CTS + coordinated MAC timing-structure, to be discussed in the next section.

\section{Coordinated directional MAC protocol (CDMAC)}

In this section, we will elaborate our MAC protocol.

\subsection{Timing-structure of CDMAC}

The timing structure of CDMAC is shown in Fig. 7. From the perspective of an arbitrary node, there are two periods 
Fig. 7 Structure of the proposed directional MAC

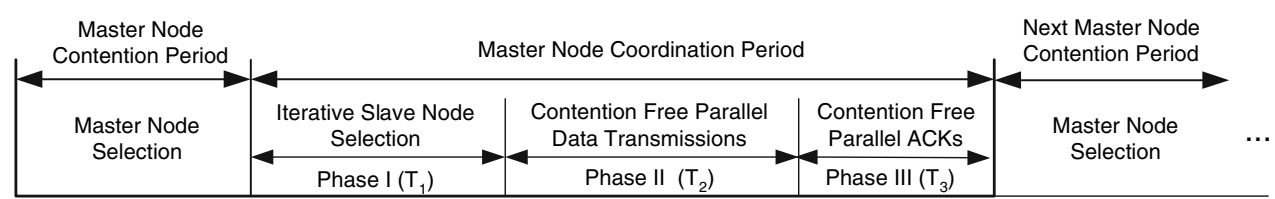

alternated locally, master-node contention period and master-node coordination period. The master-node contention period is for electing a master node-pair ${ }^{1}$ (to be defined shortly). During the master-node contention period, multiple nodes contend for channel access and the first winning node-pair is called the master node-pair. The master-node contention period ends once the master nodepair is selected. The master node-pair coordinates the activities in the following period, called master-node coordinated period, which consists of three phases. Phase I is for contention resolution of slave nodes; for this phase, an iterative collision resolution algorithm is proposed so that contending node-pairs at a later stage will not collide with the previous winning node-pairs. Phase II is for parallel collision-free DATA transmissions; in this phase, each participating node-pair may use different data rate and/or transmission power according to the channel condition; multiple DATA packet transmissions are allowed for each node-pair within the time limit of Phase II. Phase III is for parallel contention-free ACKs; with the accumulated ACK, which acknowledges all the correctly received packets sent by a node-pair in Phase II, one ACK packet is enough for each node-pair.

The proposed MAC structure takes a coordinated approach to reduce the exposed terminal problem and allow as many parallel transmissions around a selected master-node pair as possible, thus significantly increasing spatial reuse. It is worthy to note the timing-structure is local and does not require global synchronization.

\subsubsection{Length of Phase I}

The duration of the first phase of the master coordination period, $T_{1}$, can be determined by the master node-pair based on an anticipated number of non-colliding slavenode pairs and the time that is required for a node pair to exchange control messages. The anticipated number of non-colliding slave-node pairs which can transmit concurrently via direct transmission in the vicinity of the master-node pair is designated as $N$. In other words, the master-node pair plus $\mathrm{N}$ pair of slave nodes could concurrently transmit via direct transmission without causing interference to each other. The time for exchanging the control messages is designated as $T_{c t r}\left(T_{c t r}=T_{r t s}+\right.$

\footnotetext{
1 A node-pair consists of an intended data sender and an intended data receiver with certain data-transmission direction.
}

$\left.T_{c t s}+T_{c r t s}+2 S I F S+D I F S\right)$. Then $T_{1}$ can be determined according to the following expression:

$T_{1}=T_{c t r} * N * C_{1}$,

where $C_{1}$ is an adjusting parameter typically greater than one (to leave space for collision resolution). Denote $k$ as the index of the master-coordination-period determined by the current master node-pair. Following the stochastic approximation approach [23], $N^{k}$ can be iteratively updated as follows:

$$
\left\{\begin{aligned}
N^{k+1} & =\left\lceil\bar{N}^{k}+\delta^{k} \frac{\left(\bar{T}_{\text {busy }}^{k}-N^{k} T_{c t r}\right)}{T_{r t s}}\right\rceil \\
\bar{N}^{k} & =\left(1-\delta^{k}\right) \bar{N}^{k-1}+\delta^{k} N^{k} \\
\bar{T}_{\text {busy }}^{k} & =\left(1-\delta^{k}\right) \bar{T}_{\text {busy }}^{k-1}+\delta^{k} T_{\text {busy }}^{k} \\
\delta^{k} & =\left\{\begin{array}{ll}
\frac{1}{k}, & k \leq W \\
\frac{1}{W}, & k>W
\end{array},\right.
\end{aligned}\right.
$$

where $\delta^{k}$ is the adjusting step size and $T_{\text {busy }}^{k}$ is the total channel busy-time perceived by the master sending node during Phase I in master-coordination-period $k$.

\subsubsection{Length of Phase II and Phase III}

The master-node pair can determine the duration of the second phase of the master coordination period, $T_{2}$, based on a maximum size of a data packet and a minimum data rate. If the value of the former is designated as $L_{\max }$ and the value of the later is designated as $R_{\text {basic }}$, then $T_{2}$ can be determined according to the following expression:

$T_{2}=C_{2} * L_{\max } / R_{\text {basic }}$,

where $C_{2}$ is a positive integer (if it is larger than 1 , multiple packets are allowed to transmit back-to-back). The masternode pair further can determine the duration of the third phase of the master coordination period, $T_{3}$, by determining the maximum time needed to transmit an acknowledgement given the data rate, $R_{\text {basic }}$.

\section{$5.2 \mathrm{RTS} / \mathrm{CTS} / \mathrm{CRTS}$}

As mentioned in Sect. 3, we use omni RTS/CTS plus omni CRTS to reserve channel for data transmission.

The frame formats of RTS and CTS are shown in Fig. 8(a and b), respectively, which consist of original 802.11 RTS/CTS frame plus a DMAC extension. The purpose of using $802.11 \mathrm{RTS} / \mathrm{CTS}$ MAC header plus a 


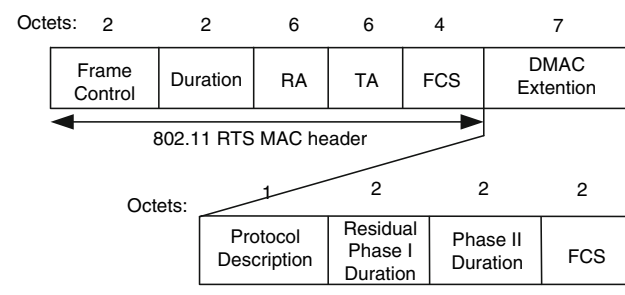

(a)

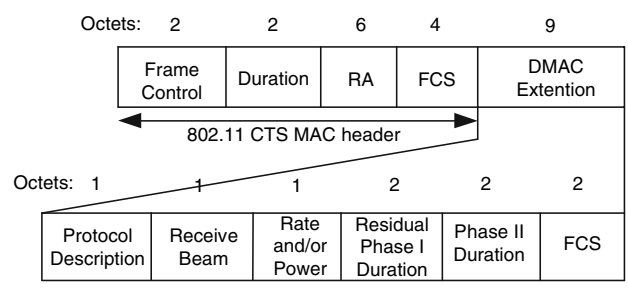

(b)

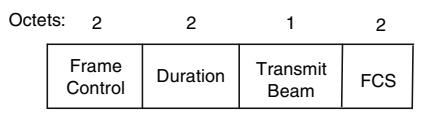

(c)

Fig. 8 (a) RTS frame format; (b) CTS frame format; (c) CRTS frame format

DMAC extension is to make newly developed directional MAC compatible with omnidirectional IEEE 802.11 MAC (We will further discuss the compatibility issue later). RTS and CTS are transmitted in the same or less rate as that used to exchange routing request message. The "duration" field included in the RTS equals the minimal time needed to finish O-RTS/O-CTS/O-CRTS. The "duration" field included in CTS equals the total time from receiving CTS to the end of current master-node-coordination period. The "residual Phase I duration" field in DMAC extension equals the residue time for slave-node contention; master nodes set this field as $T_{1}$; slave nodes will adjust this value such that each intended slave node will stop channel contention before Phase II period starts. The "Phase II duration" field in DMAC extension equals $T_{2}$. The "receive beam" field in the CTS DMAC extension indicates the beam to be used for DATA receiving (we assume each receiver can determine the angle-of-arrival (AoA) of RTS and choose appropriate receive-beam); any neighbor hearing CTS should reserve the receive-beam and guarantee the same beam will not be used for its own DATA receiving. The "rate and/or power" field in the CTS DMAC extension is the rate and/or power suggested by the receiver for data transmission; the receiver determines the appropriate rate and/or power according to channel gain and antenna gain.

The frame format of CRTS is shown in Fig. 8(c). The "duration" included in the CRTS equals the total time from receiving the CRTS to the end of current master-nodecoordination period. The "transmit beam" in the CRTS indicates the beam to be used for DATA transmission (the opposite beam of the "receive-beam" indicated in the CTS); any neighbor hearing the CRTS should reserve the transmit-beam and guarantee the same beam will not be used for its own DATA transmission.

\subsection{Master-node contention}

Following some collision-resolution algorithm (e.g., Exponential backoff algorithm or $p$-persistent algorithm), an intended sender may send omni RTS when channel is idle. The RTS includes the information fields which define the timing-structure of intended master-node coordinated period. All nodes other than the intended receiver will record the three duration values contained in the RTS and update network-allocation-vector (NAV) [1]. Upon receiving the RTS, the intended receiver can determine which receive-beam should be used based on the RTS angle-of-arrival. In addition, according to measured channel gain (based on RTS signal) and directional antenna gain, the receiver can choose appropriate rate and/or appropriate power for DATA transmission. With SIFS after receiving RTS , the intended receiver will reply an omni CTS to the intended sender, which includes the receivebeam number and rate/power value.

Upon receiving the CTS correctly, the intended sender sets appropriate transmit-beam and rate/power, whereby DATA will be transmitted in Phase II (see Fig. 7). All other nodes hearing the CTS will record the receive-beam and three duration values contained in the CTS. With SIFS after receiving CTS, the intended node will send a CRTS to reserve the transmit-beam. Now the intended node-pair become the master node pair; all other nodes hearing CTS or CRTS become slave nodes and can access the medium progressively without potential collision with the DATA transmission of the master node-pair in Phase II (see Fig. 7).

\subsection{Slave-node contention}

Slave node contention phase, i.e., Phase I, starts immediately after master-node's CRTS. Some collision-resolution algorithm like exponential backoff algorithm or $p$-persistent backoff algorithm is used for contention resolution. Similar to master-node contention, omni RTS/CTS/CRTS is used for control handshake.

Multiple slave node-pairs may win out during Phase I. However, all the winning node-pairs including master node-pair and slave node-pairs should not collide with each other in DATA or ACK transmissions. In order to achieve that, we enforce (1) any two winning node-pairs, say, nodepair A and node-pair B, do not share a beam for data 
transmission if two sending nodes are in the one-hop omni transmission range and do not share a beam for data reception if two receiving nodes are in the one-hop transmission range, and (2) A's(B's) receiver should be kept far enough from B's(A's) transmitter to avoid side-lobe interference. To judge whether A's(B's) receiver is kept far enough from B's(A's) sender, A's(B's) receiver and sender may simply evaluate the receiving power of RTS and CTS, respectively, sent by node-pair $\mathrm{B}(\mathrm{A})$. If it is greater than certain threshold, say, $\varepsilon$, we assume it will lead to a collision; otherwise, it will not. We set $\varepsilon$ as $\left(\frac{G^{d}}{G^{s}}\right)^{\beta} \epsilon_{o}$ in our simulation, where $\varepsilon_{o}$ is the carrier sensing threshold on the receiving power in 802.11 and $\beta$ is a value either 1 or 2 . If A's(B's) receiver is in the main-lobe of B's(A's) sender (illustrated in Fig. 6(b)), $\beta$ equals 1; otherwise (illustrated in Fig. 6(a)), $\beta$ equals 2.

To be clear, when a slave node (a sender) intends to contend for the channel, it first checks whether its intendto-transmit-beam (the intended sender will estimate which beam direction an intended receiver is located at based on cached information) has been reserved by the previous winning master/slave senders or whether it is too close to previous winning master/slave receivers (these information will be updated and recorded each time when a new slave node-pair wins); if not, it then omnidirectionally sends RTS. After receiving RTS, the intended slave receiver will check whether its intend-to-receive-beam has been reserved by the previous wining master/slave receivers or whether it is too close to previous winning master/slave senders; if not, the intended slave receiver will respond with a CTS that includes the receive-beam and suggested rate/power; then the intended slave sender will send a CRTS to reserve the transmit-beam.

\subsubsection{Address mobility}

In a highly mobile case, the intend-to-use-beam information cached in the intended sender may be inaccurate due to the mobility of either the sender or the receiver. If so, two cases may happen: (1) the actual receive-beam has already been reserved by a previous winning master/slave receivers; (2) the actual receive-beam is still available. If it is the first case, the slave receiver will respond with a negative CTS by setting receive-beam field to "11XXXXXX", in which the first 2 bits indicate that the beam has been already reserved and the last 6 bits indicate the actual receive-beam, whereby the sender can update the cached intend-to-use-beam information. After receiving the negative CTS, the intended sender will not continue to send CRTS. If the actual receive-beam is still available, the receive-beam field in CTS is directly set to the receivebeam number. If the intended sender receives the positive
CTS, it will continue to send CRTS to reserve the transmitbeam.

\subsection{Contention-free parallel data transmission and ACK}

In this section, we discuss DATA and ACK transmissions during Phase II and Phase III in the master-node-coordination period.

At the beginning of Phase II, each winning sender will transmit as many packets in a burst as can by using the suggested power (if power control is incorporated) and/or rate (if rate adaptation is incorporated), which was fed back by CTS. More discussions on power control, auto rate and burst transmission can be found in [15, 18, 22].

To provide link reliability, ACK is needed after DATA transmission, just as 802.11. Fortunately, only one ACK is enough if we use the accumulated ACK mechanism, i.e., one ACK acknowledges all the packets of a node-pair transmitted in Phase II.

\subsection{Compatibility with IEEE 802.11 MAC}

In this section, we examine the issue of the backwardcompatibility of our protocol with the IEEE 802.11 MAC, which is based on omnidirectional antennas.

In the master node contention period, all the nodes including omnidirectional-antenna-limited nodes and directional-antenna-capable nodes can participate in contention. In Sect 5.3, we already addressed the case in which both the intended sender and intender receiver of the master node-pair are directional-antenna capable. Now we discuss three other cases: (1) the master sending node is omnidirectional-antenna limited while the master receiving node is directional-antenna capable, (2) the master receiving node is omnidirectional-antenna limited while the master sending node is directional-antenna capable, and (3) both the master node-pair are omnidirectional-antenna limited. We assume any directionalantennas-capable node knows the antennas-capability of its intended neighbor after the neighbor discovery. In all these three cases, the master node pair will exchange RTS and CTS of the same frame format as defined in the IEEE 802.11 protocol. In order to identify the three cases, any other intended sending node needs to check whether there is a DMAC extension in RTS or CTS sent from the master node-pair. If not, other nodes should keep silent for the duration indicated in the RTS or CTS. For all the three cases, the master sending node should immediately start the transmission of DATA (i.e., Phase II) upon receiving a CTS. 
Since our scheme can address all the possible four cases as discussed above, our protocol enables an 802.11 node to transparently communicate with another directionalantenna-capable node in the same frequency band, and vice versa. Due to the popularity of 802.11 , backward compatibility is critical to the success of a MAC protocol. Our scheme solves the compatibility problem and can make use of directional antennas whenever possible.

\section{Performance evaluation and discussions}

In this section, we evaluate the performance of our protocol via ns2 simulation (Version 2.28). Simulation results are compared with DMAC [7, 8, 15] and 802.11. We did extensive simulations to study the impacts of various factors on the end-to-end throughput such as traffic load, network density, mobility, the number of antenna elements and side-lobe.

\subsection{Simulation settings}

All simulations are with a 25 node ad hoc network. The nodes are placed randomly in a 2-D square area of varying size depending on the density parameter. For all of results presented in the network, 25 UDP streams are originated, one per node, with the destination chosen randomly. We use the new random waypoint mobility model in ns-2.28. AODV routing is applied in our simulation, in which the routing discovery packets are broadcasted in the MAC layer. It is worthy to note that, for data communication, CDMAC does not need additional neighbor discovery mechanism to locate the beam direction of a mobile neighbor while DMAC may need such neighbor discovery algorithm as the one proposed in [15] or omni-direcitonal beacons to determine the beam direction of an intended receiver. To be simple, we assume each node in DMAC knows the beam location of an intended neighbor without overhead by a "God" neighbor discovery mechanism; due to this simplification, all the results regarding DMAC provided in the following figures could be worse if the overhead of neighbor discovery in real system is accounted for.

Some simulation parameters are shown in Table 2. For fair comparison, we compare CDMAC with DMAC and 802.11 using single packet transmission in the simulations although CDMAC scheme does provide multi-frame burst transmission mode for further improving efficiency. Focusing on single-packet transmission (rather than multiframe burst transmission) will allow us to validate whether CDMAC can really increase throughput by the more efficient use of directional antennas over DMAC. All the
Table 2 Simulation parameters

\begin{tabular}{llll}
\hline Parameter & Value & Parameter & Value \\
\hline PHY header & $192(\mu \mathrm{s})$ & Packet size & 1,000 (bytes) \\
Time_slot & $20(\mu \mathrm{s})$ & ACK & 112 (bits) \\
SIFS & $10(\mu \mathrm{s})$ & $W$ & 100 \\
DIFS & $50(\mu \mathrm{s})$ & $C_{1}$ & 1.4 \\
RTS & $216(\mathrm{bits})$ & $C_{2}$ & 1 \\
CTS & $184(\mathrm{bits})$ & $T_{2}$ & $4,280(\mu \mathrm{s})$ \\
CRTS & $56($ bits $)$ & $T_{3}$ & $258(\mu \mathrm{s})$ \\
\hline
\end{tabular}

simulation results shown in the paper assume single packet transmission, in which packet size is 1,000 byte. The transmission rate (for both control packets and data packets) is $2 \mathrm{Mbits} / \mathrm{s}$. The power used in the directional transmission mode is $G^{o} / G^{d}$ of that under the omni mode such that the directional transmission range equals the omni transmission range but with less power and higher spatial reuse; we set $G^{o} / G^{d}$ as $1 / M$ ( $M$ is the number of the antenna elements in each node) [3] throughout the simulation. Other simulation parameters are the same as defined in ns 2.28. The default values for transmission ranges, interference ranges and carrier sensing range are $250 \mathrm{~m}$, $445 \mathrm{~m}$ and $550 \mathrm{~m}$.

For the estimation of $N$, in our simulation, we set the $\min N$ as 0 and $\max N$ as $M-1$, where $M$ is the number of antenna sectors. Given the degree of freedom for spatial division based access is the number of antenna sectors, the maximal number of parallel transmissions (including master node-pair)in one-hop neighborhood is limited to $M$, although the actual number of neighbors could be much higher than $M$ in dense networks. We know $(0, M-1)$ is a loose bound for $N$. A tighter bound could be customized given the type of networks running.

\subsection{Simulation results}

Figure 9 shows the throughput as a function of traffic load; in this set of simulation, we set $G^{d} / G^{s}$ as $30 \mathrm{~dB}$, set average speed as $5 \mathrm{~m} / \mathrm{s}$ and set density as 60 nodes-per-square-km (the corresponding network size is $645 \mathrm{~m} * 645 \mathrm{~m}$ ). Figure 10 shows the throughput as a function of density; in this set of simulation, we set traffic load as 25 packets/ second/stream, set $G^{d} / G^{s}$ as $30 \mathrm{~dB}$, and set average speed as $5 \mathrm{~m} / \mathrm{s}$. The reason that the throughput performance improves as the increase of node density is as follows. In our simulation, we fix the number of nodes and adjust network size according to the node density. When the node density increases, the size of the network decreases. In this case, the average number of hops also reduces. So it is reasonable that the aggregated end-to-end throughput 


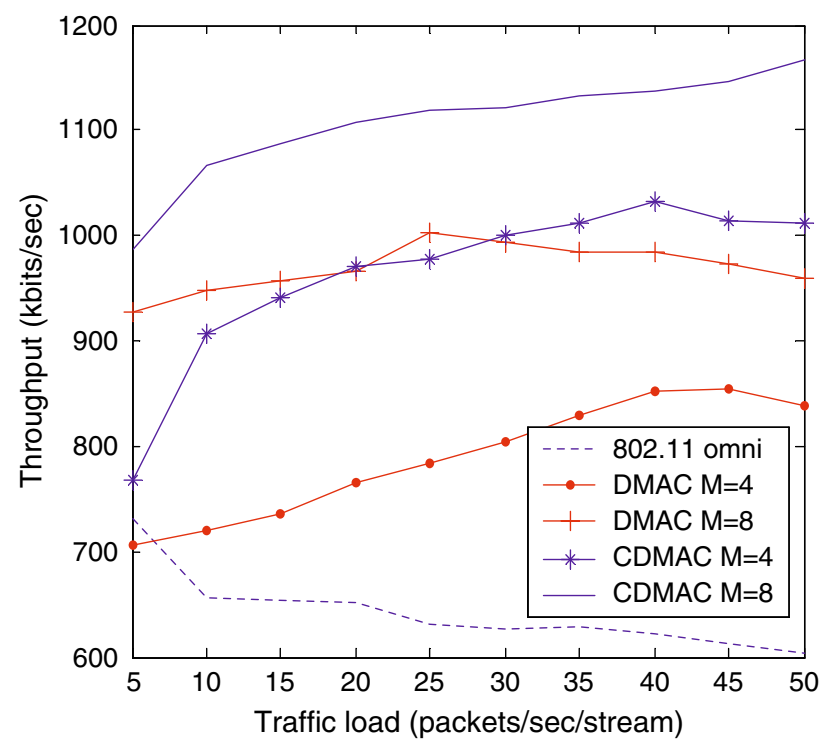

Fig. 9 Effects of traffic load

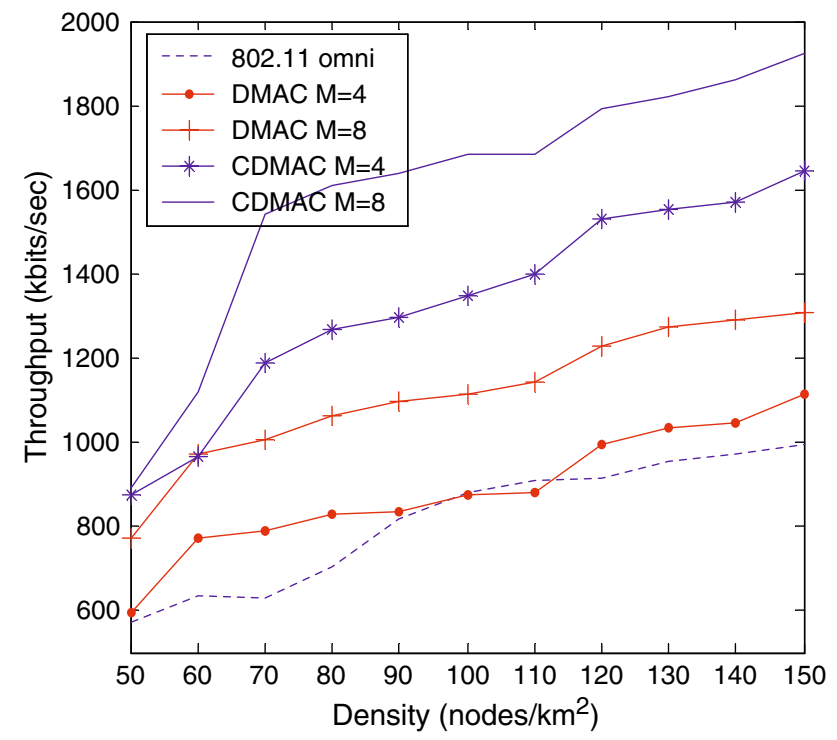

Fig. 10 Effects of density

increases even though the contention level is increased. We expect the peak will be hit around the point when every destination is within one hop away from the source.

Figure 11 shows the throughput as a function of mobility; in this set of simulation, we set traffic load as 25 packets/second/stream, set $G^{d} / G^{s}$ as $30 \mathrm{~dB}$, and set density as 80 nodes-per-square-km. Figure 12 shows the throughput as a function of main-to-side gain $\left(G^{d} / G^{S}\right)$ ratio; in this set of simulation, we set traffic load as 25 packets/second/ stream, set density as 80 nodes-per-square-km, and set average speed as $5 \mathrm{~m} / \mathrm{s}$.

Our simulation results validate what we have discussed in Sect. 4. The deafness problem, the Type I hidden terminal problem and the exposed terminal problem result in

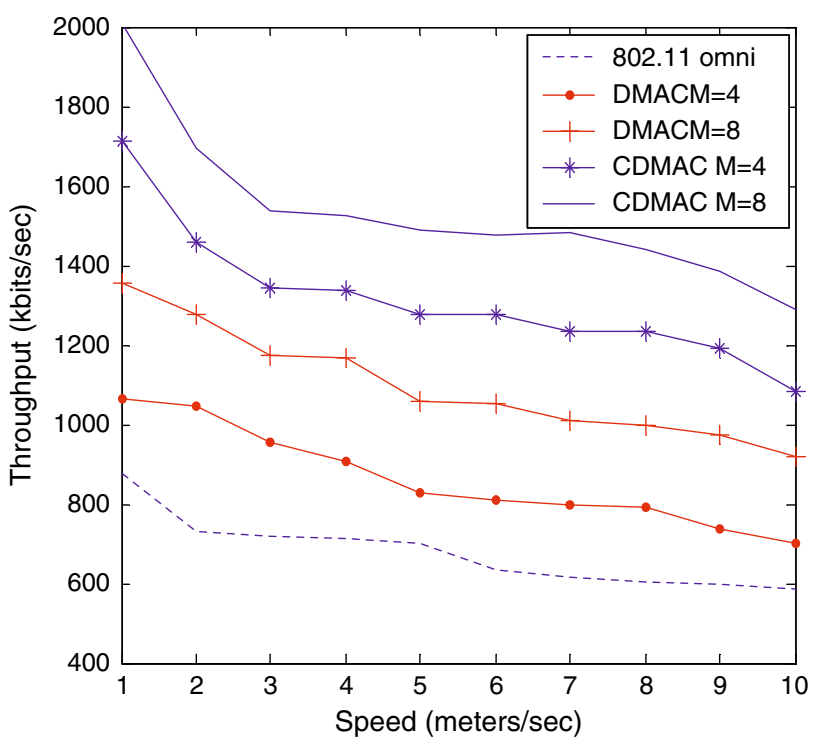

Fig. 11 Effects of mobility

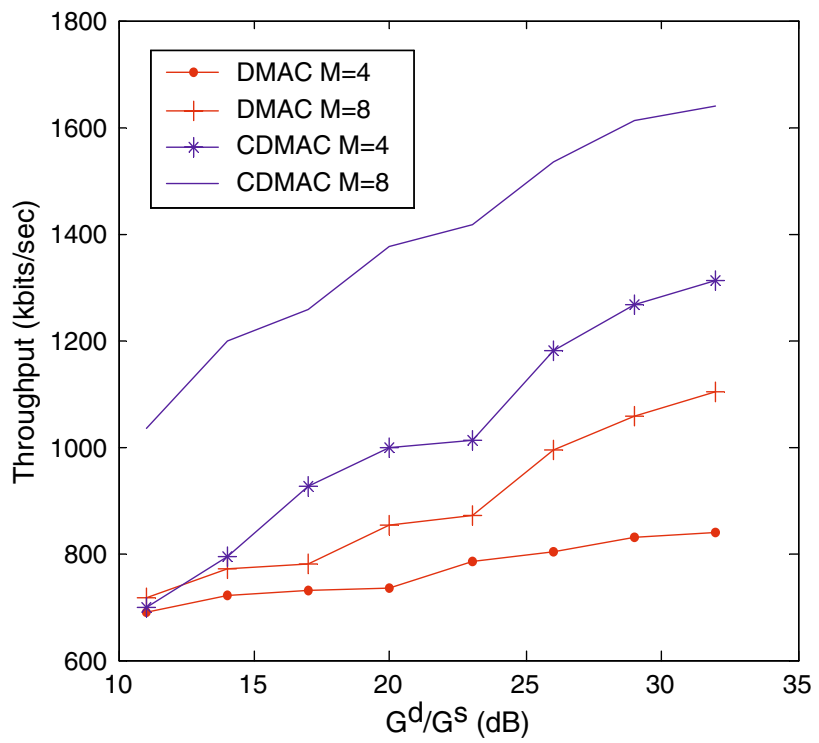

Fig. 12 Effects of side-lobe

significant performance loss under DMAC; our proposed CDMAC works much better than DMAC and 802.11 when the node density is sufficiently high (e.g., larger than 50 nodes-per-square-km) and the main-to-side gain $\left(G^{d} / G^{S}\right)$ is reasonably high (e.g., larger than $17 \mathrm{~dB}$ ). As indicated in Fig. 12, the main-to-side gain affects throughput significantly. The gains of DMAC and CDMAC over 802.11 are very limited when the main-to-side gain drops below $15 \mathrm{~dB}$. Interestingly, as the $G^{d} / G^{s}$ increases, the throughput of CDMAC increases much faster than DMAC. One possible reason is that CDMAC takes effective measures to address the side-lobe problem discussed in Sect. 4.4 but DMAC leaves the side-lobe problem wide open. 


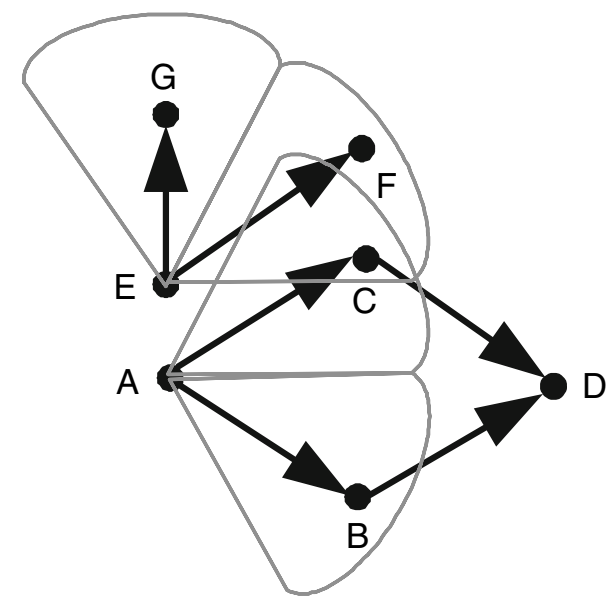

Fig. 13 An illustration of link diversity

\subsection{Further discussions}

So far, we used the first-in-first-out (FIFO) queuing service rule in the simulations presented above. The performance gain of CDMAC over 802.11 could be further improved if we can utilize the link diversity to avoid the Head-of-Line (HOL) blocking problem [20, 21]. For example, as shown in Fig. 13, when node A captures the channel in the first place for intended transmission to node $\mathrm{C}$, node $\mathrm{E}$ can still contend for channel to transmit data to node $\mathrm{G}$ rather than node F. However, the channel will be underutilized if the FIFO queuing service rule is applied and the HOL packet of node $\mathrm{E}$ is destined to node $\mathrm{F}$. We will investigate the HOL blocking problem in the future.

The last part we would mention is the limitation of our proposed scheme. As the simulation suggests, the proposed scheme works very well for dense and relatively static networks. This benefits mainly from improving spatial reuse (i.e., parallel directional transmissions) and alleviating deafness problem, exposed terminal problem and hidden terminal problem, among others. However, in sparse networks where transmission range is more important than spatial reuse, our scheme may not be suitable. Moreover, our proposed handshakes (RTS/CTS/CRTS) are omni, which is not desired in some networks, e.g., military networks, where directionality should be always preserved to keep LPI/LPD.

\section{Conclusions}

In this paper, we identified the key problems in the design of MAC protocol with directional antennas for ad hoc networks. To address these problems, we proposed a novel directional MAC protocol, called coordinated directional medium access control (CDMAC). Our CDMAC can alleviate the deafness problem, the hidden terminal problem and the exposed terminal problem with favorably low signalling overhead. Finally, it achieves high throughput and maintains backward compatibility with the IEEE 802.11 .

Acknowledgements This work was supported in part by the U.S. National Science Foundation under grants CNS-0721744 and DBI0529012. The work of Fang was also supported in part by the National Science Council (NSC), R.O.C., under the NSC Visiting Professorship with contract number NSC-96-2811-E-002-010 and Chunghwa Telecom M-Taiwan Program M-Taoyuan Project.

\section{References}

1. IEEE standard for wireless LAN medium access control (MAC) and physical layer (PHY) specifications, ISO/IEC 8802-11: 1999(E), August 1999.

2. Rappaport, T. (2002). Wireless communications principles and practice. Prentice Hall.

3. Zhang, Z., Iskander, M. F., Yun, Z., \& Madsen, A. H. (2003). Hybrid smart antenn system using directional elements-performance analysis in flat Rayleigh fading. IEEE Transactions on Antennas and Propagation, 51(10).

4. Ko, Y. B., Shankarkumar, V., \& Vaidya, N. H. (2000). Medium access control protocols using directional antennas in ad hoc networks. In Proceedings of IEEE INFOCOM.

5 Nasipuri, A., Ye, S., You, J., \& Hiromoto, R. E. (2000). A MAC protocol for mobile ad hoc networks using directional antennas. In Proceedings of the WCNC.

6. Ramanathan, R. (2000). On the performance of ad hoc networks with beamforming antennas. In Proceedings of the ACM MobiHoc, Long Beach, CA.

7. Takai, M., Martin, J., Ren, A., \& Bagrodia, R. (2002). Directional virtual carrier sensing for directional antennas in mobile ad hoc networks. In Proceedings of ACM MOBIHOC, Lausanne, Switzerland.

8. Choudhury, R. R., Yang, X., Ramanathan, R., \& Vaidya, N. (2002). Using directional antennas for medium access control in ad hoc networks. In Proceedings of ACM MOBICOM, Atlanta, Georgia.

9. Korakis, T., Jakllari, G., \& Tassiulas, L. (2003). A MAC protocol for full exploitation of directional antennas in ad-hoc wireless networks. In Proceedings of ACM Mobihoc, Annapolis, Maryland.

10. Gossain, H., Cordeiro, C. M., Cavalcanti, D., \& Agrawal, D. P. (2005). MDA: An efficient directional MAC scheme for wireless ad hoc networks. In Proceedings of IEEE GLOBECOM, St. Louis, Missouri, USA.

11. Choudhury, R. R., \& Vaidya, N. (2004). Deafness: A MAC problem in ad hoc networks when using directional antennas. In Proceedings of IEEE ICNP, Berlin.

12. Fahmy, N. S., \& Todd, T. D. (2004). A selective CSMA protocol with cooperative nulling for ad hoc networks with smart antennas. In Proceedings. of IEEE WCNC, Atlanta.

13. Singh, H., \& Singh, S. (2004). Tone based MAC protocol for use with adaptive array antennas. In Proceedings of IEEE WCNC, Atlanta.

14. Sundaresan, K., \& Sivakumar, R. (2004). A unified MAC layer framework for ad hoc networks with smart antennas. In Proceedings of ACM MobiHoc, Roppongi, Japan.

15. Ramanathan, R., Redi, J., Santivanez, C., Wiggins, D., \& Polit, S. (2005). Ad hoc networking with directional antennas: A complete system solution. Journal of Selected Areas in Communications. 
16. Muqattash, A., \& Krunz, M. (2004). A single-channel solution for transmission power control in wireless ad hoc networks. In Proceedings of MobiHoc, Tokyo, Japan.

17. Acharya, A., Misra, A., \& Bansal, S. (2003). MACA-P : A MAC for concurrent transmissions in multi-hop wireless networks. In Proceedings of the first IEEE PerCom 2003 conference, pp. 505-508.

18. Sadeghi, B., Kanodia, V., Sabharwal, A., \& Knightly, E. (2002). Opportunistic media access for multirate ad hoc networks. In Proceedings of MOBICOM.

19. Zhu, H., \& Cao, G. (2005). rDCF: A relay-enabled medium access control protocol for wireless ad hoc networks. In Proceedings of IEEE INFOCOM.

20. Wang, J., Zhai, H., \& Fang, Y. (2004). Opportunistic packet scheduling and media access control for wireless LANs and multihop ad hoc networks. In Proceedings of IEEE WCNC, Atlanta.

21. Santivanez, C., \& Redi, J. (2003). On the use of directional antennas for sensor networks. In Proceedings of IEEE MILCOM, Boston.

22. Lu, K., Wang, J., Wu, D., \& Fang, Y. (2006). Performance of a burst-frame-based CSMA/CA protocol for high data rate ultrawideband networks: Analysis and enhancement. In Proceedings of the Third International Conference on Quality of Service in Heterogeneous Wired/Wireless Networks, Waterloo, Ontario, Canada.

23. Kushner, H., \& Yin, G. (1997). Stochastic approximation algorithms and applications. New York: Springer-Verlag.

\section{Author Biographies}

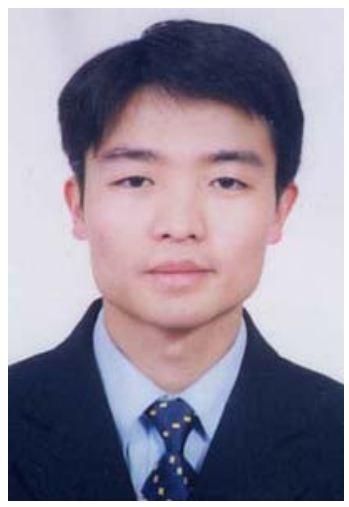

Jianfeng Wang received the B.E. and M.E. degrees in Electrical Engineering from Huazhong University of Science and Technology, China, in 1999 and 2002, respectively, and the $\mathrm{Ph} . \mathrm{D}$. degree in Electrical Engineering from University of Florida in 2006. From January 2006 to July 2006, he was a research intern in the Wireless Standards and Advanced Technology group at Intel Corporation. In October 2006, he joined Philips Research North America as a senior member research staff in the Department of Wireless Communications and Networking. He is a member of the IEEE and the ACM.

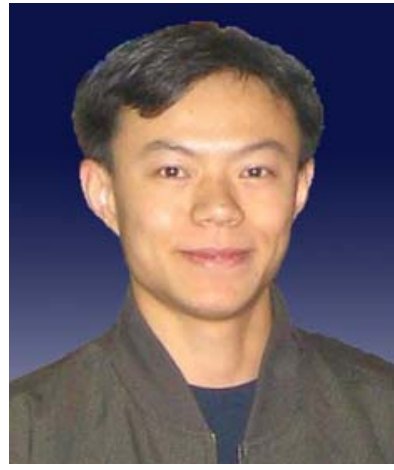

Hongqiang Zhai received the $\mathrm{Ph} . \mathrm{D}$. degree in Electrical and Computer Engineering from University of Florida in August 2006 and the B.E. and M.E. degrees in Electrical Engineering from Tsinghua University, Beijing China, in July 1999 and January 2002, respectively. He is now a senior member of research staff in Wireless Communications and Networking Department of Philips Research North America. He is a recipient of the Best Paper Award at the 14th IEEE International Conference on Network Protocols (ICNP 2006). His research interests include performance analysis, medium access control, and cross-layer design in wireless networks. $\mathrm{He}$ is a member of the $\mathrm{ACM}$ and the IEEE.

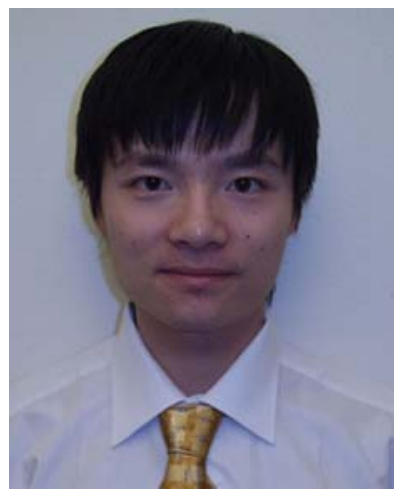

Pan $\mathbf{L i}$ received his $\mathrm{BS}$ in Electrical Engineering from Huazhong University of Science and Technology in 2005. He is working towards his Ph.D. degree in the Department of Electrical and Computer Engineering at the University of Florida. His research interests include medium access control, routing algorithms, and performance analysis in wireless networks. He is a student member of IEEE.

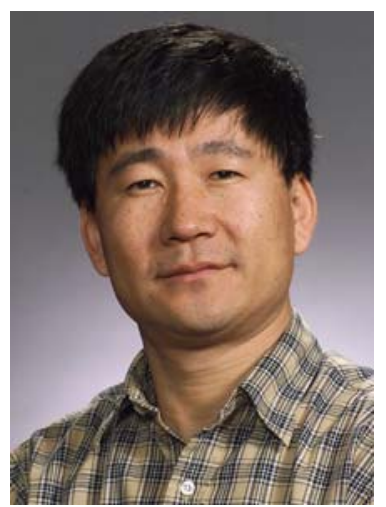

Yuguang "Michael" Fang received a Ph.D. degree in Systems, Control and Industrial Engineering from Case Western Reserve University in January 1994 and a Ph.D degree in Electrical Engineering from Boston University in May 1997.

He held a post-doctoral position in Department of Electrical and Computer Engineering at Boston University from June 1994 to August 1995. From June 1997 to July 1998, he was a Visiting Assistant Professor in Department of Electrical Engineering at the University of Texas at Dallas. From July 1998 to May 2000, he was an Assistant Professor in the Department of Electrical and Computer Engineering at New Jersey Institute of Technology. In May 2000, he joined the Department of Electrical and Computer Engineering at University of Florida, Gainesville, Florida, where he got early promotion to Associate Professor with tenure in August 2003 and to Full Professor in August 2005. He holds a University of Florida Research Foundation (UFRF) Professorship from 2006 to 2009. His research interests span many areas including wireless networks, mobile computing, mobile communications, wireless security, automatic control, and neural networks. He has published over 100 papers in refereed professional journals and over 100 papers in refereed professional conferences. He received the National Science Foundation Faculty Early Career Award in 2001 and the Office of Naval Research Young Investigator Award in 2002. He is the recipient of the Best Paper Award in IEEE International Conference on Network Protocols (ICNP) in 2006 and the recipient of the IEEE TCGN Best Paper Award in the IEEE High-Speed Networks Symposium, IEEE Globecom in 2002.

Dr. Fang has actively engaged in many professional activities. He is a Fellow of IEEE and a member of ACM. He is an Editor for several journals including IEEE Transactions on Communications, IEEE Transactions on Wireless Communications, IEEE Transactions on Mobile Computing, ACM Wireless Networks and Journal of Computer Science and Technology, and a Technical Editor for IEEE Wireless Communications Magazine. He was also an Editor for IEEE Journal on Selected Areas in Communications: Wireless Communications Series, an Area Editor for ACM Mobile Computing and Communications 
Review, an Editor for Wireless Communications and Mobile Computing, and Feature Editor for Scanning the Literature in IEEE Personal Communications. He also served on the Technical Program Committee in many professional conferences such as ACM MobiCom'02 (Committee Co-Chair for Student Travel Award), MobiCom'01, IEEE INFOCOM'07, INFOCOM'06, INFOCOM'05 (Vice-Chair for Technical Program Committee), INFOCOM'04, INFOCOM'03, INFOCOM'00, INFOCOM'98, IEEE WCNC'04, WCNC'02, WCNC'00 (Technical Program Vice-Chair), WCNC'99, IEEE Globecom'04 (Symposium Co-Chair), Globecom' 02, and International Conference on Computer Communications and Networking (IC3N) (Technical Program Vice-Chair).

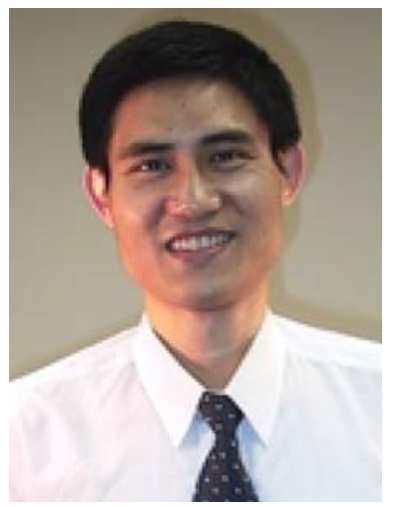

Dapeng Oliver Wu received B.E. in Electrical Engineering from Huazhong University of Science and Technology, Wuhan, China, in 1990, M.E. in Electrical Engineering from Beijing University of Posts and Telecommunications, Beijing, China, in 1997, and Ph.D. in Electrical and Computer Engineering from Carnegie Mellon University, Pittsburgh, PA, in 2003.

Since August 2003, he has been with Electrical and Computer Engineering Department at University of Florida, Gainesville, FL, as an Assistant Professor. His research interests are in the areas of networking, communications, multimedia, signal processing, and information and network security. He received the IEEE Circuits and Systems for Video Technology (CSVT) Transactions Best Paper Award for Year 2001.

Currently, he is an Associate Editor for IEEE Transactions on Wireless Communications, IEEE Transactions on Circuits and Systems for Video Technology, IEEE Transactions on Vehicular Technology, and International Journal of Ad Hoc and Ubiquitous Computing. He is also a guest-editor for IEEE Journal on Selected Areas in Communications (JSAC), Special Issue on Cross-layer Optimized Wireless Multimedia Communications. He served as Program Chair for IEEE/ACM First International Workshop on Broadband Wireless Services and Applications (BroadWISE 2004); and as a technical program committee member of over 30 conferences. He is Vice Chair of Mobile and wireless multimedia Interest Group (MobIG), Technical Committee on Multimedia Communications, IEEE Communications Society. He is a member of the Best Paper Award Committee, Technical Committee on Multimedia Communications, IEEE Communications Society. 